\title{
How Does Language Impact the Learning of Mathematics? Let Me Count the Ways
}

\author{
Geneviève Boulet \\ Mount Saint Vincent University
}

\begin{abstract}
The role that language plays in the teaching and learning of mathematics is one of some importance in the current literature in mathematics education. In this paper, I give particular attention to the manner in which teachers engage students in the exploration of mathematical concepts and procedures with the goal of revealing how language impacts students' learning. Through a series of examples of language commonly used in the mathematics classroom, I address specific issues pertaining to language used to describe mathematical processes, to read and interpret notation, and to define mathematical terms. Considering that communication is a key factor in the building of understanding, it is hoped that these examples will motivate teachers to examine and adapt their own practices in order to cultivate productive and meaningful mathematical discourse in their classrooms.
\end{abstract}

The room was filled with grade five teachers. The presenter of the workshop on teaching geometry asked the audience to take a few minutes and to write the definition of a polygon. The discomfort level arose as the minutes ticked by. It was clear the audience was becoming uncomfortable. He verified that the teachers were indeed teaching grade five geometry and that they were undertaking the study of polygons with their students. After letting some time go by, he asked for volunteers to offer their definition of a polygon. With the very first definition, avid discussions ensued, with teachers offering examples and counterexamples. All of a sudden, I found myself transported to Imre Lakatos' (1976) imaginary classroom.

After a brief introduction to the topic of the book, Lakatos depicts a classroom environment in which students attempt to gain an understanding of a particular mathematical principle (namely, the Euler theorem, though, as the appendices show, there is nothing necessarily unique about this problem). Generally, the students represent a particular approach to mathematical heuristics or a particular important individual within the history of mathematics, As conjectures are supplied, proofs are proposed, and counterexamples are offered, the students in the class gain a better understanding both of the conjecture

Geneviève Boulet is an Associate Professor of mathematics education at Mount Saint Vincent University in Halifax, Nova Scotia. For the past twenty years she has taught mathematics methods courses for preservice teachers at the elementary and secondary levels. She designs and coordinates mathematics education graduate programmes for practicing teachers. Her research focuses on the learning of rational number concepts, the impact of language on learning mathematics, and on numeracy in the workplace. 
in question and of the ways in which the conjecture can be demonstrated; indeed, the students gain a better understanding of the art of demonstration itself. (Belanger, n.d., p. 1)

It was after participating in this workshop that I began to explore the connections between the learning of mathematics and teachers' language. The grade five teachers had struggled, argued, and finally quit their attempts to define a polygon. The obstacle was language, so they said. In fact, they claimed that they knew what it was, but could not define it in words. I was puzzled and so was the presenter of the workshop. "Swearwords apart, I too am sceptical about your conception of mathematics as 'an existentially languageless activity of the mind'. How can an activity be true or false? Only articulated thought can try for truth" (Lakatos, 1976, p. 52). Can the teachers know what a polygon is and yet be incapable of defining it? After much probing and the study of an assortment of two-dimensional shapes, it became apparent that they did not know exactly what a polygon is. At first, they mistakenly accepted both intersecting polygons and open figures as simple polygons. However, with direct intervention from the presenter, the discussion progressed, their language became clearer and their growing understanding became evident. There was much more than a difficulty in finding the proper vocabulary at play here. There was an intimate relationship between their knowledge of polygons and their words to talk about them. This sparked my interest in thinking more deeply about the language that teachers use in the mathematics classroom and how it impacts the learning of mathematics.

Although Lakatos (1976) was arguing against a particular philosophical stance-mathematical formalism-he provides an example of how language is at the heart of mathematical activity. Lakatos' insights are particularly interesting at this juncture because, as Radford (2003) stresses, we are currently living though a paradigm of language: "The question is now to grasp the possibilities of discourse and to understand man as homo dialogicus. It seems that the age of Judgment inaugurated by Kant arrives to its end and we now enter into the age of Communication” (p.124). In the field of mathematics education, this increased awareness of the need to account for the connection between mathematics and language is unmistakable.

Since the 1990s, the number of studies that focus on the language of mathematics has been on the rise. As Cobb and his colleagues (1997) note: "The current reform movement in mathematics education places considerable emphasis on the role that classroom discourse can play in supporting students' conceptual development” (p. 258). Studies on the role of language in the teaching and learning of mathematics focus primarily on mathematical discourse. Some are concerned with the development of methodological frameworks to analyse mathematics discourse in the classroom (Cobb et al., 1997; Krussel, Springer \& Edwards, 2004; Ryve, 2006; Sfard, 2001) while others are concerned with the development of approaches that aim to facilitate the reading and the writing of mathematics (Barwell, Leung, Morgan \& Street, 2002; Esty, 1992; Adams, 2003; Usiskin, 1996).

Researchers in mathematics education are in agreement that communication is essential to the learning of mathematics (Ryve, 2004). Specifically, "from the perspective of mathematics learning, by articulating the principles, concepts and 
rationale behind the steps of a particular problem solution, students have the opportunity to reinforce and deepen their understanding of higher-level knowledge structures in mathematics content" (Huang, Normandia \& Greer, 2005, p.45). However, as Ryve (2004) cautions, the issue is not whether or not mathematics is learned through communicating, it is with the establishment of means that will cultivate mathematically productive discourses. While researchers grapple with the question of what constitutes productive discourses, it is important to begin supporting teachers in developing an awareness of the diverse forms of mathematical communication and how these contribute to their students' learning (Barwell et al., 2005, p. 145). Rubenstein and Thompson (2002) emphasize that "to be more aware of, and sensitive to, issues of mathematical language acquisition and to be more creative and persistent in finding ways to support children's learning, teachers must first understand children's difficulties in making sense of mathematical language” (p. 107).

Through a series of examples teachers have shared with me in my work as a mathematics educator, I address issues raised by the language that teachers use to engage pupils in classroom explorations of mathematical concepts and procedures. The purpose here is not to exhaust all manners in which they talk about mathematics; rather, my aim is to provide specific illustrations that will encourage teachers to examine more carefully the language they use in the classroom. I selected examples that reflect common practices and that serve to typify the discordant translations between formal mathematical language and natural language. In particular, I address issues that pertain to language used by teachers to describe mathematical processes, to read and interpret notation, and to define mathematical terms.

\section{Language and Mathematical Processes}

This first example addresses the issue that arises when teachers describe processes rather than explain ideas. In the following situation, Maxwell, a Grade 5 pupil, explains how he performs long division to solve the problem $148 \div 3$ : " 3 gazinta 12, 4 times; take away 12 from the 14 and bring down the 8; then 3 gazinta 28, 9 times...." After completing the operation, Maxwell asks the teacher: "What does gazinta mean?" "Actually, it is not gazinta, it is goes into," explains the teacher. "Ah", says Maxwell; "you mean it slams into it like cars do in a car crash?” Clearly, for Maxwell, the language here was not at all referring to the concepts that coordinate the steps involved in the long division algorithm. When I present this example to my student-teachers, I ask them to guess the teacher's answer to Maxwell's last question. After some thought, they generally respond that the teacher probably explained that " 3 goes into 12 , 4 times because $3+3+3+3=12$." I then ask them to explain how this string of additions can help Maxwell understand the steps of the division algorithm. In other words, why are we performing repeated addition to solve a division problem? In fact, what is a division problem? They usually have no answers to these questions other than they were told to follow those steps. Since the focus here is on describing a procedure, it is difficult to relate the long division algorithm to either a quotitive division context (the dividend is grouped into fixed quantities to learn of the number of recipients) or a partitive one (the dividend is shared equally among a fixed number of recipients to learn of the size of the portions). Yet, according to 
Squire and Bryant (2002), “children begin to solve division problems by relying on their mental model of the problem, which is built up from a schema of action that depends on the context” (p. 463). In this example, the language describing the steps of the long division algorithm is cryptic and impedes meaningful problem solving. Maxwell is not considering the intent of the division problem and is most likely unable to make sense of the answer he obtains. Simple changes to the way teachers talk in the course of performing a long division problem could make all the difference in a child's understanding. In adopting a language that sets the division problem in context, Maxwell would have a chance at making sense of the mathematics he is learning. If the teacher chose a context, either quotitive or partitive, to perform the long division problem, the steps involved in the algorithm would become comprehensible. Indeed, as Lee (2007) reiterates, "traditional algorithms can be meaningfully taught if students have opportunities to engage in conceptually sound activities and to appreciate the meaning of algorithms at the early stage, instead of relying on the mechanical memorization” (p. 48).

Division is not the only arithmetical operation that is usually presented as a recipe for children to follow. In the addition algorithm, for instance, rarely do teachers specify the nature of the unit that is being added. For example, in solving the problem $123+146$, teachers usually describe the process as follows: "First you add the 3 and the 6; that gives you 9; then you add the 2 and the 4; that gives you 6; and finally, you add the two ones together to get 2; the answer is 269." "When the procedural aspect of computation is overemphasized without clear conceptual understanding of the place value system, students tend not to think about the meaning of what they are doing and simply parrot someone else's directions in order to perform calculations” (Lee, 2007, p.48). In this example, how can the teacher be sure the student will see that the ones are added together, then the tens, and finally the hundreds?

Similarly, the teaching of the algorithms for subtraction and multiplication tend to involve the description of the procedure rather than the meaning of each of the steps, and this as concisely as possible. As Kaput (1988) states: "The language of mathematics is both a means of communication and an instrument of thought” (p. 167). Consequently, although the goal of developing an algorithm is to automate a process so that no thinking is required, students first need to learn how they are developed and how they function before they can engage fully in mathematical inquiry. This means that along with engaging students in meaningful mathematical activities, it is important for teachers to use clear language that reveals the reasoning behind mathematical procedures.

\section{Language and Mathematical Notation}

The symbolic nature of mathematics is one of the most evident features of mathematics (Pimm, 1987). In the context of learning and teaching mathematics, Pimm stresses that "... it is important to be able to distinguish at will between the symbol and the concept, between the signifier (the symbol) and the signified (the referent)” (p. 139). In fact, Pimm strongly promotes Mason's (1980) view that symbols act as windows through which we access mathematical concepts. Yet, all too often teachers express notation rather than the concepts they refer to when reading or talking about mathematics. A case in point is the language commonly 
used to talk about rational numbers. Referring to fractions as "a over b” instead of "a out of b", as in "two over three" instead of "two out of three", focuses attention on how two-thirds is written symbolically rather than on the quantity referred to by the number. With this language, students tend to view fractions as two independent whole numbers separated by a bar. Consequently, it is not surprising that when students are asked to illustrate a fraction presented to them symbolically, they produce two distinctive quantities, one to illustrate the numerator and the other to illustrate the denominator (Boulet, 1998a). In fact, in that study, the fourth graders not only illustrated each numeral, they also illustrated the fraction bar using an object such as a ruler or a pipe cleaner. In expressing the notation rather than the number represented by the notation, the very "rationality" of the number is lost. Addressing notation rather than the ideas referred to by the notation renders mathematical discourse superficial and disconnected. As Sfard and Kieran (2001) caution: "In mathematical communication, in which there are no familiar visible objects likely to serve as a basis for an attended focus, people avail themselves of symbolic artefacts as a replacement. As communication mediators, however, the symbolic substitutes are not as readily effective as the familiar objects used in everyday discourse" (p. 72). Yet, language that simply describes symbolic representations is not uncommon in the mathematics classroom. In fact, it is so frequent that teachers may have difficulty letting it go. For example, in the following excerpt of a discussion I had with a teacher about an alternative algorithm to solve fraction division problems - one that involves transforming the fractions so that they share a common denominator before dividing - the teacher's language reveals his need to perform on the symbols rather than to solve the problem using the quantities, and this in spite of the simplicity of the division problem:

G.: "Once the fractions share a common denominator, the division
problem is just like a whole number division. For example, if
you divide two thirds by one third, you need to determine how
many thirds are in two thirds, and we know that it takes two.
Having the same denominator is what makes this easy to
solve."
"So, once you have a common denominator, you just have to
divide the numerators and the denominators?"
G.: "There is no need to do that; you can simply count the number
of thirds there are in two thirds."
T.: "But, to count them, don't you have to divide the numerators
and then the denominators?"
G.: "No, you simply count the number of thirds there are in two-
thirds."

This way of talking about mathematics by talking about the symbols and how to treat them makes it difficult to make sense of mathematical ideas. Indeed, looking at the problem from a different perspective sheds light on how challenging it is to rely on notation to make sense of the mathematics: consider the case of programming computers to read mathematics aloud for visually impaired students. Hayes (1996) writes: "A text-to-speech system is likely to stumble badly when trying to read such an expression. For one thing, the usual 
left-to-right convention of English fails here; in some places within the equation the natural sequence is from top to bottom, and elsewhere from bottom to top. Furthermore, reading the symbols in any fixed sequence yields a phonetic string so long that you've forgotten the beginning by the time you reach the end" (p.110). In fact, Hayes claims that "the most mathematical of machines cannot accommodate the language of mathematics" (p. 112). Reading mathematical texts should be similar to reading ordinary texts, in that the reader must transcend the code and comprehend the text's meaning.

When the language describes the symbols, the teacher must teach rules to coordinate the connection of one notation to another or of one procedure to another. Consider for example, the case of decimal numbers. Teachers who refer to 0.37 by mentioning each symbol, zero point three seven, and not as the number thirty-seven hundredths, are then obligated to teach a rule to connect this number to its equivalent as a percentage: "Move the decimal over two places to the right." However, thirty-seven hundredths is thirty-seven percent; there is no need to perform any kind of operation on the symbols, such as moving the decimal point, to view the number in decimal notation as a percentage. In fact, moving the decimal point changes the value of the number. The only difference between the decimal number and its equivalent percentage notation is the manner in which the number is written symbolically, either as 0.37 , thirty-seven hundredths, or as $37 \%$, thirty-seven percent. To regard numbers as lists of discrete symbols may account for the difficulties students have with decimal numbers. Indeed, according to Hiebert (1985), the first site of students' difficulty to solve problems with decimal numbers is their inability to connect the symbols to their referents. These difficulties continue even into adulthood as Bonotto (2005) suggests: "Studies of pre-service elementary teachers' understanding of decimals...identified significant difficulties in interpreting decimal numbers and solving word problems involving decimals" (p. 319). Furthermore, the introduction of more and more rules to be able to connect mathematical ideas at the symbolical level becomes unmanageable and is often at the root of students' claim that mathematics is irrelevant and abstract.

Describing symbols is not the only issue that is raised by the reading of mathematical notation. In geometry, for example, students learn to study shapes and to measure different attributes of objects. More specifically, "instructional programs from pre-kindergarten through grade 12 should enable all students to understand measurable attributes of objects and the units, systems, and processes of measurement and to apply appropriate techniques, tools and formulas to determine measurements" (National Council of Teachers of Mathematics, 2000). Most school curricula take a progressive approach to learning measurement. To help children understand the purpose of measuring, activities designed in the lower grades usually focus on the physical attributes of objects and the use of informal units while those designed in the middle grades focus on the use of standard units. Finally in the higher elementary grades, students learn to generalize measurement techniques and construct formulas. It is interesting to note that great care goes into developing concepts of measurement throughout the early school years and yet, when the formulas for measuring basic shapes are introduced, much of what they learned is suddenly contradicted; or so it appears when one considers the language typically used to talk about measurement. 
Initially, students learn about attributes generally measured (time, length, degrees, etc.) Then they learn how to determine an appropriate unit of measurement to use in order to measure that attribute (from informal, unconventional units to formal, conventional units). Finally, they learn how to perform the measurement (iterating the unit of measurement and using measuring tools). However, as the following example demonstrates, this logical approach to learning measurement vanishes when measurement formulas are introduced. Consider one of the most basic formulas, the one used for calculating the surface area of a rectangle, $A=h \times b$ ("area is height times base"). For example, students learn that a $3 \mathrm{~cm}$ by $5 \mathrm{~cm}$ rectangle has a surface area of $3 \mathrm{~cm} \times 5 \mathrm{~cm}=15 \mathrm{~cm}^{2}$. The following excerpt of a discussion between a $6^{\text {th }}$ grade teacher and one of his students shows how much the language, both written and spoken, is clearly confusing to the student:

Blaise: So, a centimetre multiplied by a centimetre makes a square centimetre?

Mr. D.: That is right, because inside the rectangle, you see, it makes squares.

Blaise: So, when you multiply lengths you always get squares?

Mr. D.: Yes, that is right; and when you multiply squares you get cubes.

Blaise: But how does multiplying them make them square?

Mr. D.: Because it cuts up each side and you multiply to know how many there are.

Blaise: I just don't see how multiplying the two sides tells me how much is inside.

The language the teacher uses here is confusing in three ways, one with regards to the arithmetical operation of multiplication, another with regards to the construction of geometrical shapes, and lastly, one with regards to measurement. First, the two numbers involved in a multiplication problem, i.e., the multiplicand and the multiplier, do not count the same objects (Boulet, 1998b): One number counts the objects while the other counts the groups of objects. Consequently, on the very onset, centimetres cannot be multiplying centimetres as it is expressed both in writing and verbally. The other issue is with the claim that squares are constructed from no more than two lengths. Finally, and most importantly, the centimetre is not the appropriate unit of measurement to use to measure surface areas. Units of measurement are simply smaller versions of the attribute to measure; that is, a unit of length is used to measure a length; a unit of surface is required to measure a surface. In the above example, the square centimetre should be the unit of measurement to use, and this should be clear right from the start. Indeed, the unit of measurement should not arise out of the measuring, as it is the case in the excerpt; rather, the unit of measurement is what needs to be used to perform the measurement. The centimetres in this example measure the length of each side of the rectangle and these actually provide the rectangle's dimensions. The relationship between those dimensions and the number of square centimetres on the inside is the missing link the student is searching for. In addition to understanding the steps involved in constructing a measurement formula, it is important for teachers to initiate students in how to interpret 
symbolic notation. In other words, the formula here should be interpreted as the reflection of the measurement process rather than of the dimensions of the rectangle. That is, the number of squares in one row is given by the base, the number of rows is given by the height, and the unit of measurement is the square centimetre: $3 \times 5 \mathrm{~cm}^{2}$ (there are three rows of five square centimetres).

The preceding example demonstrates the need to support teachers in developing strategies for reading mathematical texts. As Borasai (1998) suggests: "Because students have such difficulties coping with these texts, research on reading mathematics has concentrated on developing and studying strategies that teach students the 'language of mathematics' and ways to interpret word problems...As a result, reading strategies that reflect the new concern for reading as meaning-making are rarely incorporated into mathematics instruction" (p. 276). Just as students are taught to read 123 as one hundred and twenty-three and not as one, two, three, they should be encouraged, right from the start, to interpret any mathematical expression, along with its symbols and specialised vocabulary, with a language that elucidates the expression's meaning rather than its notational form. However, that being said, it is by no means an effortless task for the teacher. As Pimm (1987) emphasizes: "Because of structural differences between mathematical and English prose text, a different style of reading needs to be adopted by the reader, and pupils need considerable training on how to read mathematics" (p. 184).

\section{Language and Mathematical Vocabulary}

It is not possible to discuss language in the mathematics classroom without addressing matters of vocabulary. The following examples consider different aspects of the teaching and learning of mathematical terms. In the quest for ways to foster mathematical discourse, it is important for teachers not to go astray and be content with just any kind of talk or, on the contrary, to be content with just a specific kind of talk. Although this appears to be common sense, it may not be easy to identify which talk is too imprecise and which is too restrictive. For example, it would be problematic to let students continue to speak of "round things" when speaking of "circles", but it should be acceptable to let them say "take away" instead of "subtract." In an attempt at removing ambiguities and strengthening pupils' mathematical vocabulary, some teachers may at times inadvertently promote language that takes the "natural" out of the "natural language." Consider this example of a discussion between a seventh grade teacher and one of her pupils about the language to use to talk about whole and decimal numbers:

Mrs. Smith: The word 'and' is only used to indicate a decimal point, as in twenty and five-tenths (20.5).

Sophie: $\quad$ So, how can that work when I say 'three hundred and five'?"

Mrs. Smith: You cannot say that; you have to say 'three hundred five', the 'and' is always a decimal point.

Sophie: But, we always say 'and' with just whole numbers, like when we talk about the date, like two thousand and six. 
Mrs. Smith: I know, but we shouldn't; we must say two thousand six.

Introducing rules like this one that modify the usage of natural language may make mathematics appear artificial and discourage students from engaging in mathematical discourse. It is undeniable that speaking about mathematics can sometimes lead to ambiguities. For example, in saying two hundred and threethousandths, we could be speaking about either 200.003 or 0.203 . However, according to Byers (2007), "ambiguous situations contain the potential for change; they are dynamic and can be creative. Ambiguity points to a valid way of thinking involved in mathematics, one that needs to find its place alongside logic if we are to account for the power and effectiveness of the discipline” (p. B12). Questioning students as to how they could be clearer in their language rather than imposing a rule that modifies natural language would lead to interesting discussions about the rigour of mathematics and how best to communicate mathematical information.

Learning vocabulary requires learning definitions. According to Morgan (2005), "the choice of a particular definition is presented both in relation to general community values (transparency) and as a personal or contextual matter, related to 'our intended applications'” (p. 110). This does not mean that a particular concept changes depending on the context; rather, it simply means that different perspectives or descriptions of the same concept serve different purposes at different times. For example, consider the definition of a square. At times, it could be more useful to define it in terms of its properties, e.g., opposite sides are parallel and all four angles are $90^{\circ}$, and, at other times, in terms of its classification, e.g., a rectangle whose sides are all the same length. In any case, the same concept - the square-is being defined.

This important, but subtle, distinction between the manner in which a concept is defined and the substance of the concept can make all the difference in how teachers interpret and use definitions in the classroom. For example, some teachers believe that mathematical concepts become more complex as students mature and that the definitions should therefore reflect that complexity: A Grade 11 teacher once explained to me that probabilities are defined differently in high school than they are in elementary school. In particular, she believed that probability was defined in a more primitive form-a fraction, for younger students - and that a more sophisticated definition—probability as a divisionwas reserved for older, more able students. The fact that fractions can be quotients in division problems does not transform a concept of number, the fraction, into a concept of an arithmetical operation, a division. A fraction is a rational number, the ratio between part and whole, and this regardless of the context it appears in, e.g., arithmetical operations, probabilities, measurements, etc., and regardless of the age of the learner. Here, the teacher is confusing the meaning of a concept, that of a theoretical probability, with one of the many situations which yield fractions, such as division problems.

A similar case is found in the example of teaching students that the result of a subtraction is called a difference. To impose such a definition creates confusion on many levels. Already, young children have difficulty understanding problems involving difference (Clarke, 2003). To define the term difference to mean the 
result of subtraction adds to this confusion. Children learn how objects or situations are different and how they are alike starting at an early age. Indeed, many activities for preschoolers are designed to teach them to compare and to categorize. As Lung (2005) suggests, "the teaching of technical vocabulary should be seen as a pedagogic point of departure for exploring concepts, meaning-making and meaning exchanging, not an end point of learning” (p. 134). To restrict the usage of the word difference to mean the result of a subtraction does the opposite of what Lung proposes. It does not engage students in thinking about how numbers can be different. For instance, they could be different in parity, in primality, in size, etc. Moreover, children are not usually inclined to use subtraction to determine the difference in size of two numbers; more often than not, children use the method of counting on (Clarke, 2003). As in the previous example regarding the definition of a fraction, the concept is being confused with one of its applications. The fact that subtraction is one method that can be used to determine the difference in size of two numbers does not necessarily modify the meaning of the remainder. Subtraction is the arithmetical operation that aims to determine the quantity that remains after a certain number of objects have been removed. In determining the difference in size of two numbers, one can choose to remove one quantity from the other to discover by how much they differ. In other words, the remainder can be used in a comparison situation to determine the difference in size of two numbers. In conserving the meanings of mathematical words, students have a chance at making connections across mathematical topics. For example, students who have learned that the difference is the result of subtracting often do not make the connection between the remainder in a division problem and a remainder in a subtraction problem. Indeed, my student-teachers often tell me they are quite different. For them, in the case of division, the remainder is what cannot be further divided and in subtraction it is what's left over. Even with the help of examples and a clear explanation of the connection, they do not readily accept that the remainder in division is in truth the remainder in subtraction, so convinced are they that the result of a subtraction is a difference.

\section{Conclusion}

Language plays a key role in the mathematics classroom. In fact, "fluency in it provides access to the whole world of mathematics” (Esty, 1992, p. 32). Much of the attention to mathematical discourse focuses on students' ability to communicate by clarifying and justifying their ideas and procedures (National Council of Teachers of Mathematics, 1991). However, the teacher's role in fostering productive mathematical discourse in the classroom is central. In addition to being responsible for creating the opportunities for students to engage in discussions, exploring, negotiating, and sharing knowledge (Manouchehri \& Enderson, 1999), the teacher's own use of language in the mathematics classroom serves as an important example of effective communication. The few examples presented in this paper provide tangible support for Raiker's (2002) claim that spoken language is in large part responsible for problems in the teaching and learning of mathematics. In fact, the results of that study reveal clearly that "above all, teachers must be aware of the language they use when teaching mathematics and that the recommended vocabulary...should be used 
with caution" (p. 59). As Selden and Selden (1997) emphasize, “The way teachers explain mathematics depends, to a large extent, on the conceptual grasp they acquire in their college classes. In addition, they often teach as they were taught, modeling themselves after their college mathematics teachers as much as their K-12 teachers” (p. 1). Consequently, it is not sufficient to tell teachers to be more sensitive to the language used in mathematical conversations; addressing specific instances of language commonly used in the mathematics classroom helps teachers identify more clearly what sort of language can be a source of difficulties and helps them understand why such language must be adapted in order to make it more meaningful. The goal of presenting these examples of language regularly used to describe mathematical processes, to read and interpret mathematical notation, and to define and to understand mathematical terms, is to offer teachers grounds for examining and adjusting their own practices.

\section{Acknowledgement}

I wish to thank Jeannine Maynard and Nezihe Elik for their time and helpful comments.

\section{References}

Adams, T. L. (2003). Reading mathematics: More than words can say. The Reading Teacher, 56(8), 786-795.

Barwell, R., Leung, C., Morgan. C., \& Street, B. (2002). The language dimension of mathematics teaching. Mathematics Teaching, 180, 12-15.

Barwell, R., Leung, C., Morgan, C., \& Street, B. (2005). Applied linguistics and mathematics education: More than words and numbers. Language and Education, 19(2), 142-146.

Belanger, J. (n.d.). Review of Proofs and Refutations by Imre Lakatos. Unpublished manuscript. Retrieved November 15, 2006, from http://www.owlnet.rice.edu/ phil530/JohnBBookR.pdf

Bonotto, C. (2005). How informal out-of-school mathematics can help students make sense of formal in-school mathematics: The case of multiplying by decimal numbers. Mathematical Thinking and Learning, 7(4), 313-344.

Borasi, R., Siegel, M., Fonzi, J., \& Smith, C. F. (1998). Using transactional reading strategies to support sense-making and discussion in mathematics classrooms: An exploratory study. Journal for Research in Mathematics Education, 29(3), 275-305.

Boulet, G. (1998a). Didactical implications of children's difficulties in learning the fraction concept. Focus on Learning Problems in Mathematics, 20(4), 19-34.

Boulet, G. (1998b). On the essence of multiplication. For the Learning of Mathematics, 18(3), 1219.

Byers, W. (2007). Ambiguity and paradox in mathematics. The Chronicle of Higher Education, 53(48), B12.

Clarke, D. (2003). An issue in teaching and learning subtraction: What's the difference? Australian Primary Mathematics Classroom, 8(3), 4-11.

Cobb, P., Boufi, A., McClain, K., \& Whitenack, J. (1997). Reflective discourse and collective reflection. Journal for Research in Mathematics Education, 28(3), 258-77.

Esty, W. W. (1992). Language concepts of mathematics. Focus on Learning Problems in Mathematics, 14(4), 31-54.

Hayes, B. (1996). Speaking of mathematics. American Scientist, 84(2), 110-116.

Hiebert, J. (1985). Children's knowledge of common and decimal fractions. Education and Urban Society, 17, 427-437.

Huang J., Normandia, B., \& Greer, S. (2005). Communicating mathematically: Comparison of knowledge structures in teacher and student discourse in a secondary math classroom. Communication Education, 54(1), 34-51. 
Kaput, J. J. (1988). Linking representations in the symbol systems of algebra. In S. Wagner \& C. Kieran (Eds.), Research issues in the learning and teaching of algebra, Vol. 4, (pp.167-194). Reston, VA: National Council of Teachers of Mathematics.

Krussel, L., Springer, G. T., \& Edwards, B. (2004). The teacher's discourse moves: a framework for analyzing discourse in mathematics classrooms. School Science and Mathematics, 104(7), 307-313.

Lakatos, I. (1976). Proofs and refutations. Cambridge, England: Cambridge University Press.

Lee, J.-E. (2007). Making sense of the traditional long division algorithm. The Journal of Mathematical Behavior, 26(1), 48-59.

Lung, C. (2005). Mathematical vocabulary: Fixers of knowledge or points of exploration? Language and Education, 19(2), 127-135.

Manouchehri, A. \& Enderson, M. C. (1999). Promoting mathematical discourse: learning from classroom examples. Mathematics Teaching in the Middle School, 4(4), 216.

Mason, J. (1980). When is a symbol symbolic? For the Learning of Mathematics, 1(2), 8-12.

Morgan, C. (2005). Words, definitions and concepts in discourses of mathematics, teaching and learning. Language and education, 19(2), 103-115.

National Council of Teachers of Mathematics (2000). Principles and standards for school mathematics. Reston, VA: National Council of Teachers of Mathematics.

National Council of Teachers of Mathematics (1991). Professional standards for school mathematics. Reston, VA: National Council of Teachers of Mathematics.

Pimm, D. (1987). Speaking mathematically: Communication in mathematics classrooms. London: Routledge \& Kegan Paul.

Radford, L. (2003). On the epistemological limits of language: Mathematical knowledge and social practice during the renaissance. Educational Studies in Mathematics, 52, 123-150.

Raiker, A. (2002). Spoken language and mathematics. Cambridge Journal of Education, 32(1), 4560.

Rubenstein, R. N. \& Thompson, D. R. (2002). Understanding and supporting children's mathematical vocabulary development. Teaching Children Mathematics, 9(2), 107-112.

Ryve, A. (2006). Making explicit the analysis of students' mathematical discourses-revisiting a newly developed methodological framework. Educational Studies in Mathematics, 62(2), 191209.

Ryve, A. (2004). Can collaborative concept mapping create mathematically productive discourses? Educational Studies in Mathematics, 26, 157-177.

Selden, A. \& Selden, J. (1997). Preservice teachers' conceptions of mathematics and how to teach it. Research Sampler. MAA Online: The Mathematical Association of America.

Sfard, A. (2001). There is more to discourse than meets the ears: Looking at thinking as communication to learn more about mathematical learning. Educational Studies in Mathematics, 46(1-3), 13-57.

Sfard, A. \& Kieran, C. (2001). Cognition as communication: rethinking learning-by-talking through multi-faceted analysis of students' mathematical interactions. Mind, Culture, and Activity, $8(1), 42-76$.

Squire, S. \& Bryant, P. (2002). From sharing to dividing: young children's understanding of division. Developmental Science, 5(4), 452-466.

Usiskin, Z. (1996). Mathematics as a language. In Communication in Mathematics K-12 and Beyond: 1996 Yearbook (pp. 231-243). Reston VA: National Council of Teachers of Mathematics. 